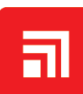 \\ CHITKARA \\ Journal of Nuclear Physics, Material Sciences, Radiation and Applications \\ Journal homepage: https://jnp.chitkara.edu.in/
}

\section{Determination of Gamma-Ray Shielding Parameters for Concretes and Dosimeters Using MCNPX}

\author{
Huseyin Ozan Tekin ${ }^{1}$ and V.P. Singh ${ }^{2}$ iD \\ ${ }^{1}$ Department of Medical Diagnostic Imaging, University of Sharjah, P.O. Box-27272, Sharjah, United Arab Emirates \\ ${ }^{2}$ Department of Physics, Karnatak University, Dharwad, Karnataka-580003, India \\ 1tekin765@gmail.com \\ 22kudphyvps@rediffmail.com (Corresponding Author)
}

\section{ARTICLE INFORMATION}

Received: April 16, 2020

Revised: October 19, 2020

Accepted: November 04, 2020

Published Online: December 03, 2020

\section{Keywords:}

Concrete, Dosimeter, Attenuation

Coefficient, Gamma radiations, MCNPX

\section{ABSTRACT}

Gamma-ray shielding parameter for some concretes and dosimeters having large scale applications in radiological protection are presented using MCNPX (version 2.4.0) at different energies. The MCNPX results are compared with experimental, MCNP and XCOM data, and good agreement is being noted. Present study indicates that MCNPX simulation method is suitable and reliable simulation tool to be used as an alternative method for the investigation of gamma-ray interaction. The present geometry can be used as standard geometry for MCNPX simulation for low- as well as high-Z materials.

DOI: $10.15415 /$ jnp. 2020.81009

\section{Introduction}

Radiation is being one of the important radiations used in medical, agriculture, industries, consumer products, archaeology, and is being found in nuclear technology. Radiation protection is branch of science and technology, emphasis is given on reduction and measurement of radiation exposure. Various types of shielding materials are being invented and investigated in detail for shielding and dosimetric application for use in radiological protection. The personal dosimeters are being used for radiation dose measurement using various types of materials based on the requirements of user and application. The tissue equivalent materials are being used for personal dosimeter to represent the realistic radiation interaction similar to human body organs. Water exhibits adequate suitability as tissue equivalent for radiation interaction and being considered most useful in medical applications for simulation purpose. Shielding and dosimetric material are characterized using mass attenuation coefficients and it's derived parameters (effective atomic number, effective electron densities, etc). The mass attenuation coefficient is a elementary factor for gamma-ray interaction parameters [1-5]. The investigations on mass attenuation coefficients using Monte Carlo simulation for shielding and dosimetric material [6-12] have been reported. Radiation (neutrons, photons and electrons) interaction with various types of material attenuation or energy deposition has been reported and is found to be an effective tool for assessment of effectiveness of shielding and dosimetric properties.

MCNPX is a general purpose radiation transport code for simulation of interaction of radiation with materials. MCNPX is three-dimensional and it utilizes the nuclear cross section libraries and uses particle physics models [13]. Capability of MCNPX Monte Carlo code and comparison with experiment results are found by Tekin et al. [14-19] in the literature. The MCNPX code is developed by the Los Alamos National Laboratory was used for simulation of gamma-ray interactions. The photon intensity i. e. number of photons per unit volume for particular energy incident on a material is being transmitted, absorbed and/or back scattered towards source.

The gamma-ray attenuation coefficient is the combination of interaction processes photoelectric absorption, Compton scattering and pair production. These partial interaction processes dependent upon photon energy and atomic number 
(Z) of the elements of the mateiral. The XCOM program is for calculation $\mathrm{X}$ - and gamma-ray attenuation coefficients for compounds or mixtures in energy $1 \mathrm{keV}-100 \mathrm{GeV}$ [20]. User friendly windows base operating system WinXcom is new version of XCOM program [21].

Concretes and dosimeters are the high demanding materials required for nuclear and radiation science and technology. The concretes are the mixture of low- as well as high- $\mathrm{Z}$ elements suitable for shielding from neutron and photon whereas dosimeter materials are low-Z materials resembling the radiological characteristics of human body organs. The concrete is found one of most important shielding materials due to special quality for shielding application, cost-effectiveness, handling, construction in desired shape and size and decommissioning. The shielding and dosimeter materials are the key materials for radiological protection for any radiation or nuclear facility.

In order to understand the interaction of photon with the shielding and dosimeter materials, it is vital to determine the mass attenuation coefficients for characterization of materials. Recently gamma-ray interaction with concretes and dosimeters has been reported using Monte Carlo simulation and experiment. In the present work, we have calculated mass attenuation coefficients for concretes and dosimeters using the MCNPX code with the objective to test the alternate simulation method. The chemical compositions of these materials are taken from literature [22-24]. The mass attenuation coefficients for of the concretes and dosimeters were calculated, and results were compared with XCOM program, experimental data [22-23] and simulation results [6-7, 11-12]. The present investigation would be very useful for providing alternate simulation tool for shielding and dosimetry in the radiation protection.

\section{Materials and Methods}

The mass attenuation coefficient $(\mu / \rho)$ values for the concretes and dosimeters are theoretically calculated by using mixture rule $\left((\mu / \rho)_{\text {polymer }}=\sum_{i}^{n} w_{i}(\mu / \rho)_{i}\right)$ where $w_{i}$ is the proportion by weight and $(\mu / \rho)_{i}$ is mass attenuation coefficient of the ith element by using XCOM Berger et al. [20]. The uncertainties in $\mu / \rho$ values is about $1 \%$ for low-Z $(1<\mathrm{Z}<8)$ in Compton region $(30 \mathrm{keV}$ to 100 $\mathrm{MeV})$. Below $30 \mathrm{keV}$ energy, the uncertainties are as much as $5-10 \%$ because of correction to experiments for high- $Z$ impurities and departure of Compton cross section from Klein-Nishina theory. Also above $100 \mathrm{MeV}$ photon energy, uncertainties in $\mu / \rho$ values may be $5-10 \%$. Uncertainties in photon energy absorption coefficient may be slightly greater values. The gamma sources of photon energies above $5 \mathrm{keV}$ are being used in medical, biological, industrial, radioactive source transportation and other shielding applications. Hence uncertainty in the result may not have any impact for practical applications. The mass attenuation coefficient $(\mu / \rho)$ values for the selected materials were calculated using MCNPX simulation code for photon energies, 59.5, 279.1, 511, 661.6, 662, 1173.2, 1274.5 and $1332.5 \mathrm{keV}$. The present results were compared with earlier investigation in addition to the theoretical XCOM and experimental results from literature.

MCNPX code is a radiation transport code used for modeling the radiation transport and the interaction of $\mathrm{X}$ - and gamma-ray, neutron and electrons radiation with the matter. It is based on the Monte Carlo method to solve the transport equation; furthermore, it can work on different modes of delivery that are capable to consider neutrons, electrons and photons, alone, or in pairs all three together. In this study, MCNPX (version 2.4.0) Monte Carlo code has been used for investigations on attenuation properties of different type of materials. MCNPX simulation parameters such as cell definitions, surface definitions, material definition and position of each tool, definitions and features of sources have been defined in input file according to their properties. The geometrical form of material samples have been defined as a cube with the sizes of $10 \mathrm{~cm}$ (height) x $10 \mathrm{~cm}$ (width) $\mathrm{x} 5 \mathrm{~cm}$ (thickness). The total simulation geometry is seen in Figure 1. The schematic view of MCNPX simulation setup with $\mathrm{Pb}$ collimator, investigated dosimeter materials samples, $\mathrm{Pb}$ shields for backscattered and unused photons and detection area have been presented in Figure 2 with defined geometries in MCNPX input file. Gamma-ray source, lead $(\mathrm{Pb})\left(\right.$ density $\left.=11,34 \mathrm{~g} / \mathrm{cm}^{3}\right)$ collimators and shields, samples and detection area have been defined in cell card, surface card and data card sections of MCNPX input. The basic variables such as CEL, ERG, DIR, POS, and PAR have been defined, respectively. The geometric center of detection area has been considered for location of point source. The source has been defined as a point source at photon energies of 59.5, 279.1, 511, 661.6, 662, 1173.2, 1274.5 and $1332.5 \mathrm{keV}$. To acquire absorbed dose amount in detection area, energy deposition mesh tally (F6) has been used. This type of tally in MCNPX scores energy deposition data in which energy deposited per unit volume from all particles is included. MCNPX calculations were completed by using Intel ${ }^{\circ}$ Core $^{\mathrm{TM}}$ i7 CPU $2.80 \mathrm{GHz}$ computer hardware. The error rate has been observed less than $\% 1$ in output file. The same simulation parameters have been applied for all samples. In each simulation, density of dosimeter materials have been defined in input file. 


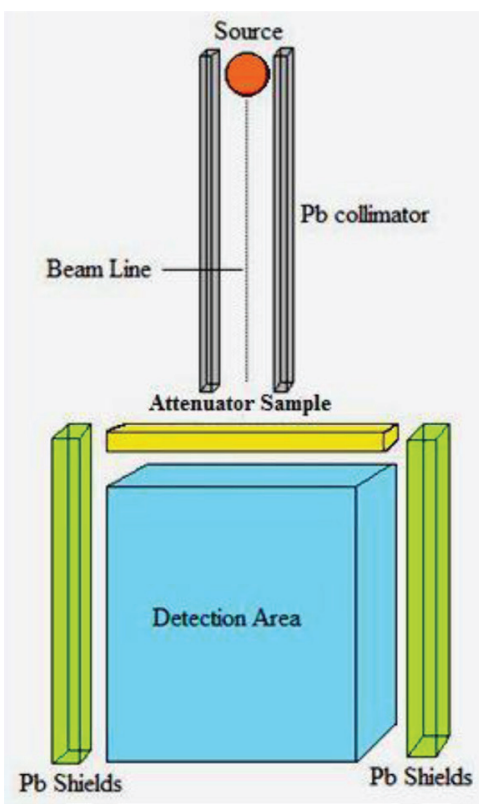

Figure 1: Total simulation geometry.

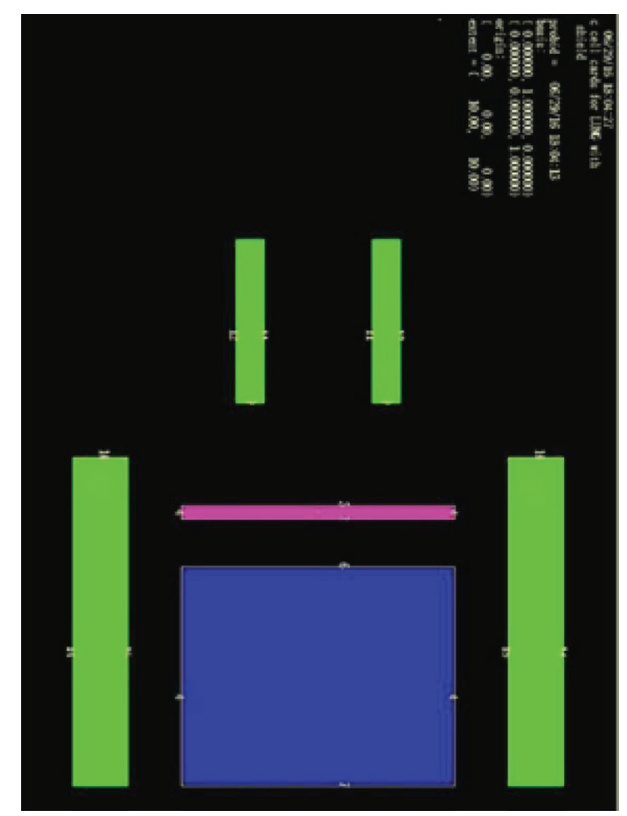

Figure 2: MCNPX simulation setup of total simulation geometry.

Table 1 (a): Mass attenuation coefficient concrete shielding materials using MCNPX and comparison with XCOM, Expt, Geant4 and MCNP.

\begin{tabular}{|c|c|c|c|c|c|c|c|c|c|c|c|}
\hline \multirow{2}{*}{$\begin{array}{r}\text { Energy } \\
(\mathrm{MeV})\end{array}$} & \multirow[b]{2}{*}{ MCNPX } & \multicolumn{4}{|c|}{ Ordinary Concrete } & \multirow{2}{*}{$\begin{array}{c}\text { Energy } \\
(\mathrm{MeV})\end{array}$} & \multirow[b]{2}{*}{ MCNPX } & \multicolumn{4}{|c|}{ Hematite-serpentine } \\
\hline & & Xcom & Exp. & Geant4 & MCNP & & & Xcom & Exp. & Geant4 & MCNP \\
\hline 1.5 & $5.04 \mathrm{E}-02$ & $5.19 \mathrm{E}-02$ & $7.13 \mathrm{E}-02$ & $6.12 \mathrm{E}-02$ & $5.15 \mathrm{E}-02$ & 1.5 & $4.96 \mathrm{E}-02$ & $5.15 \mathrm{E}-02$ & $4.96 \mathrm{E}-02$ & $5.05 \mathrm{E}-02$ & $5.29 \mathrm{E}-02$ \\
\hline 2 & 4.19E-02 & $4.47 \mathrm{E}-02$ & $5.04 \mathrm{E}-02$ & $4.88 \mathrm{E}-02$ & 4.44E-02 & 2 & 4.19E-02 & 4.46E-02 & $4.20 \mathrm{E}-02$ & $4.32 \mathrm{E}-02$ & 4.70E-02 \\
\hline 3 & $3.27 \mathrm{E}-02$ & $3.64 \mathrm{E}-02$ & $4.30 \mathrm{E}-02$ & $3.89 \mathrm{E}-02$ & $3.62 \mathrm{E}-02$ & 3 & $3.36 \mathrm{E}-02$ & $3.67 \mathrm{E}-02$ & $3.72 \mathrm{E}-02$ & $3.81 \mathrm{E}-02$ & $3.85 \mathrm{E}-02$ \\
\hline 4 & $2.73 \mathrm{E}-02$ & $3.17 \mathrm{E}-02$ & $3.78 \mathrm{E}-02$ & $3.56 \mathrm{E}-02$ & $3.15 \mathrm{E}-02$ & 4 & $2.74 \mathrm{E}-02$ & $3.24 \mathrm{E}-02$ & $3.52 \mathrm{E}-02$ & $3.30 \mathrm{E}-02$ & $3.44 \mathrm{E}-02$ \\
\hline 5 & $2.47 \mathrm{E}-02$ & $2.88 \mathrm{E}-02$ & $3.39 \mathrm{E}-02$ & $2.68 \mathrm{E}-02$ & $2.86 \mathrm{E}-02$ & 5 & $2.44 \mathrm{E}-02$ & $2.97 \mathrm{E}-02$ & $3.20 \mathrm{E}-02$ & $3.11 \mathrm{E}-02$ & $3.04 \mathrm{E}-02$ \\
\hline 6 & $2.17 \mathrm{E}-02$ & $2.68 \mathrm{E}-02$ & $3.39 \mathrm{E}-02$ & $3.20 \mathrm{E}-02$ & $2.66 \mathrm{E}-02$ & 6 & $2.27 \mathrm{E}-02$ & $2.80 \mathrm{E}-02$ & $3.28 \mathrm{E}-02$ & $3.12 \mathrm{E}-02$ & $2.76 \mathrm{E}-02$ \\
\hline \multirow{2}{*}{$\begin{array}{c}\text { Energy } \\
(\mathrm{MeV})\end{array}$} & & \multicolumn{4}{|c|}{ ilmenite-limonite } & \multirow{2}{*}{$\begin{array}{c}\text { Energy } \\
(\mathrm{MeV})\end{array}$} & \multicolumn{5}{|c|}{ Basalt-magnetite } \\
\hline & MCNPX & Xcom & Exp. & Geant4 & MCNP & & MCNPX & Xcom & Exp. & Geant4 & MCNP \\
\hline 1.5 & 4.84E-02 & $5.05 \mathrm{E}-02$ & $5.48 \mathrm{E}-02$ & $5.13 \mathrm{E}-02$ & $5.17 \mathrm{E}-02$ & 1.5 & 4.97E-02 & $5.14 \mathrm{E}-02$ & $4.56 \mathrm{E}-02$ & 4.98E-02 & $5.27 \mathrm{E}-02$ \\
\hline 2 & 4.07E-02 & $4.37 \mathrm{E}-02$ & 4.07E-02 & $4.23 \mathrm{E}-02$ & $4.36 \mathrm{E}-02$ & 2 & $4.16 \mathrm{E}-02$ & 4.44E-02 & $3.61 \mathrm{E}-02$ & $4.01 \mathrm{E}-02$ & 4.47E-02 \\
\hline 3 & $3.27 \mathrm{E}-02$ & $3.63 \mathrm{E}-02$ & $3.48 \mathrm{E}-02$ & $3.54 \mathrm{E}-02$ & $3.74 \mathrm{E}-02$ & 3 & 3.37E-02 & $3.65 \mathrm{E}-02$ & $3.11 \mathrm{E}-02$ & $3.46 \mathrm{E}-02$ & $3.80 \mathrm{E}-02$ \\
\hline 4 & $2.74 \mathrm{E}-02$ & $3.23 \mathrm{E}-02$ & $3.17 \mathrm{E}-02$ & $3.11 \mathrm{E}-02$ & $3.34 \mathrm{E}-02$ & 4 & $2.77 \mathrm{E}-02$ & $3.23 \mathrm{E}-02$ & $2.69 \mathrm{E}-02$ & $3.01 \mathrm{E}-02$ & $3.34 \mathrm{E}-02$ \\
\hline 5 & $2.47 \mathrm{E}-02$ & $2.99 \mathrm{E}-02$ & $2.97 \mathrm{E}-02$ & $3.01 \mathrm{E}-02$ & $3.03 \mathrm{E}-02$ & 5 & $2.44 \mathrm{E}-02$ & $2.96 \mathrm{E}-02$ & $2.79 \mathrm{E}-02$ & $2.88 \mathrm{E}-02$ & $3.06 \mathrm{E}-02$ \\
\hline 6 & $2.27 \mathrm{E}-02$ & $2.84 \mathrm{E}-02$ & $2.97 \mathrm{E}-02$ & $3.00 \mathrm{E}-02$ & $2.83 \mathrm{E}-02$ & 6 & $2.29 \mathrm{E}-02$ & $2.79 \mathrm{E}-02$ & & $2.52 \mathrm{E}-02$ & $2.74 \mathrm{E}-02$ \\
\hline \multirow{2}{*}{$\begin{array}{l}\text { Energy } \\
(\mathrm{MeV})\end{array}$} & \multicolumn{5}{|c|}{ ilmenite } & \multirow{2}{*}{$\begin{array}{c}\text { Energy } \\
(\mathrm{MeV})\end{array}$} & \multicolumn{5}{|c|}{ Steel-scrap } \\
\hline & MCNPX & Xcom & Exp. & Geant4 & MCNP & & MCNPX & Xcom & Exp. & Geant4 & MCNP \\
\hline 1.5 & 4.87E-02 & $5.03 \mathrm{E}-02$ & $5.71 \mathrm{E}-02$ & $5.16 \mathrm{E}-02$ & $5.30 \mathrm{E}-02$ & 1.5 & 4.96E-02 & $5.03 \mathrm{E}-02$ & $4.90 \mathrm{E}-02$ & $5.05 \mathrm{E}-02$ & $5.25 \mathrm{E}-02$ \\
\hline 2 & $4.12 \mathrm{E}-02$ & $4.36 \mathrm{E}-02$ & $4.39 \mathrm{E}-02$ & $4.30 \mathrm{E}-02$ & 4.37E-02 & 2 & $4.12 \mathrm{E}-02$ & 4.37E-02 & $5.20 \mathrm{E}-02$ & $4.81 \mathrm{E}-02$ & $4.42 \mathrm{E}-02$ \\
\hline 3 & $3.43 \mathrm{E}-02$ & $3.62 \mathrm{E}-02$ & $3.67 \mathrm{E}-02$ & $3.60 \mathrm{E}-02$ & $3.72 \mathrm{E}-02$ & 3 & $3.36 \mathrm{E}-02$ & $3.65 \mathrm{E}-02$ & $4.48 \mathrm{E}-02$ & 4.04E-02 & $3.81 \mathrm{E}-02$ \\
\hline
\end{tabular}




\begin{tabular}{cccccc}
4 & $2.89 \mathrm{E}-02$ & $3.22 \mathrm{E}-02$ & $3.28 \mathrm{E}-02$ & $3.21 \mathrm{E}-02$ & $3.24 \mathrm{E}-02$ \\
5 & $2.49 \mathrm{E}-02$ & $2.98 \mathrm{E}-02$ & $3.20 \mathrm{E}-02$ & $3.03 \mathrm{E}-02$ & $3.13 \mathrm{E}-02$ \\
6 & $2.36 \mathrm{E}-02$ & $2.83 \mathrm{E}-02$ & & $2.91 \mathrm{E}-02$ & $2.83 \mathrm{E}-02$ \\
\hline \multirow{2}{*}{$\begin{array}{c}\text { Energy } \\
(\mathbf{M e V})\end{array}$} & & & Steel-magnetite & \\
\cline { 2 - 6 } 1.5 & $4.98 \mathrm{E}-02$ & $4.98 \mathrm{E}-02$ & $4.31 \mathrm{E}-02$ & $4.65 \mathrm{E}-02$ & $5.18 \mathrm{E}-02$ \\
2 & $4.09 \mathrm{E}-02$ & $4.34 \mathrm{E}-02$ & $3.97 \mathrm{E}-02$ & $4.02 \mathrm{E}-02$ & $4.23 \mathrm{E}-02$ \\
3 & $3.29 \mathrm{E}-02$ & $3.64 \mathrm{E}-02$ & $3.60 \mathrm{E}-02$ & $3.71 \mathrm{E}-02$ & $3.63 \mathrm{E}-02$ \\
4 & $2.88 \mathrm{E}-02$ & $3.29 \mathrm{E}-02$ & $3.52 \mathrm{E}-02$ & $3.30 \mathrm{E}-02$ & $3.32 \mathrm{E}-02$ \\
5 & $2.56 \mathrm{E}-02$ & $3.09 \mathrm{E}-02$ & $3.41 \mathrm{E}-02$ & $3.20 \mathrm{E}-02$ & $3.20 \mathrm{E}-02$ \\
6 & $2.38 \mathrm{E}-02$ & $2.97 \mathrm{E}-02$ & & $2.88 \mathrm{E}-02$ & $2.90 \mathrm{E}-02$ \\
\hline
\end{tabular}

$\begin{array}{llllll}4 & 2.98 \mathrm{E}-02 & 3.28 \mathrm{E}-02 & 3.95 \mathrm{E}-02 & 3.68 \mathrm{E}-02 & 3.29 \mathrm{E}-02 \\ 5 & 2.63 \mathrm{E}-02 & 3.06 \mathrm{E}-02 & 4.30 \mathrm{E}-02 & 4.04 \mathrm{E}-02 & 3.08 \mathrm{E}-02 \\ 6 & 2.48 \mathrm{E}-02 & 2.92 \mathrm{E}-02 & & 2.81 \mathrm{E}-02 & 2.92 \mathrm{E}-02\end{array}$

Table 1 (b): Mass attenuation coefficient dosimetric materials using MCNPX and comparison with XCOM, Expt and Geant4.

\begin{tabular}{|c|c|c|c|c|c|c|c|c|c|}
\hline \multirow{2}{*}{$\begin{array}{l}\text { Energy } \\
(\mathrm{MeV})\end{array}$} & \multicolumn{4}{|c|}{ LiF } & \multirow{2}{*}{$\begin{array}{c}\text { Energy } \\
(\mathrm{MeV})\end{array}$} & \multicolumn{4}{|c|}{$\mathrm{C}_{4} \mathrm{H}_{6} \mathrm{BaO}_{4}$} \\
\hline & MCNPX & $\mathrm{XCOM}$ & Expt & Geant4 & & MCNPX & XCOM & Expt & Geant4 \\
\hline 0.2792 & 7.31E-02 & $1.02 \mathrm{E}-01$ & $1.01 \mathrm{E}-01$ & $9.70 \mathrm{E}-02$ & 0.2792 & $1.76 \mathrm{E}-01$ & $1.68 \mathrm{E}-01$ & $1.65 \mathrm{E}-01$ & $1.61 \mathrm{E}-01$ \\
\hline 0.3201 & $6.98 \mathrm{E}-02$ & $9.66 \mathrm{E}-02$ & $9.50 \mathrm{E}-02$ & $9.00 \mathrm{E}-02$ & 0.3201 & $1.41 \mathrm{E}-01$ & $1.43 \mathrm{E}-01$ & $1.41 \mathrm{E}-01$ & $1.33 \mathrm{E}-01$ \\
\hline 0.514 & $5.70 \mathrm{E}-02$ & 7.98E-02 & $8.01 \mathrm{E}-02$ & $7.10 \mathrm{E}-02$ & 0.514 & $7.76 \mathrm{E}-02$ & $9.38 \mathrm{E}-02$ & $9.28 \mathrm{E}-02$ & $8.80 \mathrm{E}-02$ \\
\hline 0.6616 & 4.97E-02 & $7.15 \mathrm{E}-02$ & $7.11 \mathrm{E}-02$ & $6.80 \mathrm{E}-02$ & 0.6616 & $6.05 \mathrm{E}-02$ & 7.92E-02 & $7.91 \mathrm{E}-02$ & $7.40 \mathrm{E}-02$ \\
\hline 1.115 & $3.63 \mathrm{E}-02$ & $5.57 \mathrm{E}-02$ & $5.55 \mathrm{E}-02$ & $5.20 \mathrm{E}-02$ & 1.115 & $3.96 \mathrm{E}-02$ & $5.82 \mathrm{E}-02$ & $5.83 \mathrm{E}-02$ & $5.50 \mathrm{E}-02$ \\
\hline 1.173 & $3.51 \mathrm{E}-02$ & $5.45 \mathrm{E}-02$ & $5.37 \mathrm{E}-02$ & $5.10 \mathrm{E}-02$ & 1.173 & $3.80 \mathrm{E}-02$ & $5.68 \mathrm{E}-02$ & $5.71 \mathrm{E}-02$ & $5.10 \mathrm{E}-02$ \\
\hline 1.333 & $3.22 \mathrm{E}-02$ & $5.11 \mathrm{E}-02$ & $5.06 \mathrm{E}-02$ & $4.80 \mathrm{E}-02$ & 1.333 & $3.44 \mathrm{E}-02$ & $5.31 \mathrm{E}-02$ & $5.29 \mathrm{E}-02$ & $4.90 \mathrm{E}-02$ \\
\hline \multirow{2}{*}{$\begin{array}{c}\text { Energy } \\
(\mathrm{MeV}) \\
\end{array}$} & \multicolumn{4}{|c|}{$\mathrm{CdSO}_{4}$} & \multirow{2}{*}{$\begin{array}{c}\text { Energy } \\
(\mathrm{MeV})\end{array}$} & \multicolumn{4}{|c|}{$\mathrm{SrSO}_{4}$} \\
\hline & MCNPX & XCOM & Expt & Geant4 & & MCNPX & XCOM & Expt & Geant4 \\
\hline 0.2792 & $1.42 \mathrm{E}-01$ & $1.45 \mathrm{E}-01$ & $1.43 \mathrm{E}-01$ & $1.42 \mathrm{E}-01$ & 0.2792 & $1.04 \mathrm{E}-01$ & $1.22 \mathrm{E}-01$ & $1.21 \mathrm{E}-01$ & $1.23 \mathrm{E}-01$ \\
\hline 0.3201 & $1.17 \mathrm{E}-01$ & $1.26 \mathrm{E}-01$ & $1.25 \mathrm{E}-01$ & $1.20 \mathrm{E}-01$ & 0.3201 & $9.18 \mathrm{E}-02$ & $1.11 \mathrm{E}-01$ & $1.10 \mathrm{E}-01$ & $1.08 \mathrm{E}-01$ \\
\hline 0.514 & $7.13 \mathrm{E}-02$ & $8.86 \mathrm{E}-02$ & 8.72E-02 & $8.10 \mathrm{E}-02$ & 0.514 & $6.37 \mathrm{E}-02$ & $8.48 \mathrm{E}-02$ & $8.46 \mathrm{E}-02$ & 8.00E-02 \\
\hline 0.6616 & $5.82 \mathrm{E}-02$ & 7.63E-02 & $7.60 \mathrm{E}-02$ & $7.10 \mathrm{E}-02$ & 0.6616 & $5.39 \mathrm{E}-02$ & 7.46E-02 & 7.41E-02 & $7.50 \mathrm{E}-02$ \\
\hline 1.115 & $3.96 \mathrm{E}-02$ & $5.72 \mathrm{E}-02$ & 5.72E-02 & $5.00 \mathrm{E}-02$ & 1.115 & $3.79 \mathrm{E}-02$ & $5.71 \mathrm{E}-02$ & $5.73 \mathrm{E}-02$ & $5.10 \mathrm{E}-02$ \\
\hline 1.173 & $3.80 \mathrm{E}-02$ & $5.58 \mathrm{E}-02$ & $5.63 \mathrm{E}-02$ & $5.10 \mathrm{E}-02$ & 1.173 & $3.65 \mathrm{E}-02$ & $5.59 \mathrm{E}-02$ & $5.61 \mathrm{E}-02$ & $5.00 \mathrm{E}-02$ \\
\hline 1.333 & $3.46 \mathrm{E}-02$ & $5.22 \mathrm{E}-02$ & $5.24 \mathrm{E}-02$ & $5.30 \mathrm{E}-02$ & 1.333 & $3.34 \mathrm{E}-02$ & 5.23E-02 & $5.25 \mathrm{E}-02$ & $5.40 \mathrm{E}-02$ \\
\hline \multirow{2}{*}{$\begin{array}{c}\text { Energy } \\
(\mathrm{MeV})\end{array}$} & \multicolumn{4}{|c|}{$\mathrm{CaSO}_{4}$} & \multirow{2}{*}{$\begin{array}{c}\text { Energy } \\
(\mathrm{MeV})\end{array}$} & \multicolumn{4}{|c|}{$\mathrm{CaCO}_{3}$} \\
\hline & MCNPX & XCOM & Expt & Geant4 & & MCNPX & XCOM & Expt & Geant4 \\
\hline 0.2792 & $8.50 \mathrm{E}-02$ & $1.12 \mathrm{E}-01$ & $1.12 \mathrm{E}-01$ & $1.09 \mathrm{E}-01$ & 0.2792 & $8.53 \mathrm{E}-02$ & $1.12 \mathrm{E}-01$ & $1.11 \mathrm{E}-01$ & $1.01 \mathrm{E}-01$ \\
\hline 0.3201 & 7.99E-02 & $1.06 \mathrm{E}-01$ & $1.05 \mathrm{E}-01$ & $1.04 \mathrm{E}-01$ & 0.3201 & $7.98 \mathrm{E}-02$ & $1.06 \mathrm{E}-01$ & $1.04 \mathrm{E}-01$ & $1.00 \mathrm{E}-01$ \\
\hline 0.514 & $6.30 \mathrm{E}-02$ & 8.67E-02 & $8.62 \mathrm{E}-02$ & $8.10 \mathrm{E}-02$ & 0.514 & $6.28 \mathrm{E}-02$ & 8.67E-02 & $8.59 \mathrm{E}-02$ & $8.40 \mathrm{E}-02$ \\
\hline 0.6616 & $5.50 \mathrm{E}-02$ & 7.75E-02 & 7.77E-02 & 7.30E-02 & 0.6616 & $5.48 \mathrm{E}-02$ & 7.75E-02 & 7.72E-02 & $7.10 \mathrm{E}-02$ \\
\hline 1.115 & 3.97E-02 & $6.03 \mathrm{E}-02$ & $6.05 \mathrm{E}-02$ & $6.50 \mathrm{E}-02$ & 1.115 & $3.95 \mathrm{E}-02$ & $6.03 \mathrm{E}-02$ & $6.05 \mathrm{E}-02$ & $6.30 \mathrm{E}-02$ \\
\hline 1.173 & $3.83 \mathrm{E}-02$ & $5.90 \mathrm{E}-02$ & $5.91 \mathrm{E}-02$ & $5.30 \mathrm{E}-02$ & 1.173 & $3.81 \mathrm{E}-02$ & $5.90 \mathrm{E}-02$ & $5.91 \mathrm{E}-02$ & $5.90 \mathrm{E}-02$ \\
\hline 1.333 & $3.50 \mathrm{E}-02$ & $5.52 \mathrm{E}-02$ & $5.53 \mathrm{E}-02$ & $5.80 \mathrm{E}-02$ & 1.333 & 3.49E-02 & $5.52 \mathrm{E}-02$ & $5.54 \mathrm{E}-02$ & $5.10 \mathrm{E}-02$ \\
\hline
\end{tabular}




\begin{tabular}{|c|c|c|c|c|c|c|c|c|c|}
\hline \multirow{2}{*}{$\begin{array}{c}\text { Energy } \\
(\mathrm{MeV})\end{array}$} & \multicolumn{4}{|c|}{$\mathrm{BaSO}_{4}$} & \multirow{2}{*}{$\begin{array}{c}\text { Energy } \\
(\mathrm{MeV})\end{array}$} & \multicolumn{4}{|c|}{$3 \mathrm{CdSO}_{4} .8 \mathrm{H}_{2} \mathrm{O}$} \\
\hline & MCNPX & XCOM & Expt & Geant4 & & MCNPX & XCOM & Expt & Geant4 \\
\hline 0.2792 & $1.78 \mathrm{E}-01$ & $1.71 \mathrm{E}-01$ & $1.68 \mathrm{E}-01$ & $1.76 \mathrm{E}-01$ & 0.2792 & $1.37 \mathrm{E}-01$ & $1.40 \mathrm{E}-01$ & $1.38 \mathrm{E}-01$ & $1.29 \mathrm{E}-01$ \\
\hline 0.3201 & $1.41 \mathrm{E}-01$ & $1.44 \mathrm{E}-01$ & $1.42 \mathrm{E}-01$ & 1.49E-01 & 0.3201 & $1.15 \mathrm{E}-01$ & $1.24 \mathrm{E}-01$ & $1.23 \mathrm{E}-01$ & $1.13 \mathrm{E}-01$ \\
\hline 0.514 & 7.75E-02 & $9.24 \mathrm{E}-02$ & $9.00 \mathrm{E}-02$ & $9.30 \mathrm{E}-02$ & 0.514 & 7.07E-02 & $9.00 \mathrm{E}-02$ & $8.90 \mathrm{E}-02$ & $9.10 \mathrm{E}-02$ \\
\hline 0.6616 & $6.12 \mathrm{E}-02$ & $7.75 \mathrm{E}-02$ & 7.75E-02 & 7.20E-02 & 0.6616 & $5.76 \mathrm{E}-02$ & $7.80 \mathrm{E}-02$ & 7.79E-02 & 7.10E-02 \\
\hline 1.115 & $4.00 \mathrm{E}-02$ & $5.65 \mathrm{E}-02$ & $5.64 \mathrm{E}-02$ & $5.10 \mathrm{E}-02$ & 1.115 & $3.93 \mathrm{E}-02$ & $5.90 \mathrm{E}-02$ & $5.91 \mathrm{E}-02$ & $5.00 \mathrm{E}-02$ \\
\hline 1.173 & $3.86 \mathrm{E}-02$ & $5.52 \mathrm{E}-02$ & $5.54 \mathrm{E}-02$ & 5.10E-02 & 1.173 & $3.78 \mathrm{E}-02$ & $5.76 \mathrm{E}-02$ & $5.81 \mathrm{E}-02$ & $5.20 \mathrm{E}-02$ \\
\hline 1.333 & $3.46 \mathrm{E}-02$ & $5.15 \mathrm{E}-02$ & $5.16 \mathrm{E}-02$ & $5.40 \mathrm{E}-02$ & 1.333 & $3.46 \mathrm{E}-02$ & $5.39 \mathrm{E}-02$ & 5.37E-02 & 4.80E-02 \\
\hline \multirow{2}{*}{$\begin{array}{c}\text { Energy } \\
(\mathrm{MeV})\end{array}$} & \multicolumn{4}{|c|}{$\mathrm{CaSO}_{4} .2 \mathrm{H}_{2} \mathrm{O}$} & \multirow{2}{*}{$\begin{array}{c}\text { Energy } \\
(\mathrm{MeV})\end{array}$} & \multicolumn{4}{|c|}{ Perspex } \\
\hline & MCNPX & XCOM & Expt & Geant4 & & MCNPX & XCOM & Expt & Geant4 \\
\hline 0.2792 & $8.48 \mathrm{E}-02$ & $1.14 \mathrm{E}-01$ & $1.13 \mathrm{E}-01$ & $1.08 \mathrm{E}-01$ & 0.2792 & $8.42 \mathrm{E}-02$ & $1.18 \mathrm{E}-01$ & - & $1.48 \mathrm{E}-01$ \\
\hline 0.3201 & 7.97E-02 & $1.08 \mathrm{E}-01$ & $1.07 \mathrm{E}-01$ & $1.00 \mathrm{E}-01$ & 0.3201 & 7.99E-02 & $1.12 \mathrm{E}-01$ & - & $1.44 \mathrm{E}-01$ \\
\hline 0.514 & $6.30 \mathrm{E}-02$ & $8.86 \mathrm{E}-02$ & 8.84E-02 & $8.90 \mathrm{E}-02$ & 0.514 & $6.42 \mathrm{E}-02$ & $9.30 \mathrm{E}-02$ & -- & $9.78 \mathrm{E}-02$ \\
\hline 0.6616 & $5.50 \mathrm{E}-02$ & 7.92E-02 & $8.33 \mathrm{E}-02$ & $7.60 \mathrm{E}-02$ & 0.6616 & $5.62 \mathrm{E}-02$ & $8.33 \mathrm{E}-02$ & - & $8.26 \mathrm{E}-02$ \\
\hline 1.115 & $3.97 \mathrm{E}-02$ & $6.16 \mathrm{E}-02$ & $6.19 \mathrm{E}-02$ & $6.90 \mathrm{E}-02$ & 1.115 & 4.06E-02 & $6.50 \mathrm{E}-02$ & -- & 7.19E-02 \\
\hline 1.173 & $3.84 \mathrm{E}-02$ & $6.03 \mathrm{E}-02$ & $6.05 \mathrm{E}-02$ & $6.20 \mathrm{E}-02$ & 1.173 & $3.99 \mathrm{E}-02$ & $6.36 \mathrm{E}-02$ & -- & 7.23E-02 \\
\hline 1.333 & $3.51 \mathrm{E}-02$ & $5.65 \mathrm{E}-02$ & $5.62 \mathrm{E}-02$ & $5.40 \mathrm{E}-02$ & 1.333 & $3.61 \mathrm{E}-02$ & $5.95 \mathrm{E}-02$ & -- & $6.02 \mathrm{E}-02$ \\
\hline \multirow{2}{*}{$\begin{array}{c}\text { Energy } \\
(\mathrm{MeV})\end{array}$} & \multicolumn{4}{|c|}{ Alanine } & \multirow{2}{*}{$\begin{array}{c}\text { Energy } \\
(\mathrm{MeV}) \\
\end{array}$} & \multicolumn{4}{|c|}{ Bakelite } \\
\hline & MCNPX & XCOM & Expt & Geant4 & & MCNPX & XCOM & Expt & Geant4 \\
\hline 0.2792 & $8.28 \mathrm{E}-02$ & $1.18 \mathrm{E}-01$ & -- & $1.62 \mathrm{E}-01$ & 0.2792 & $8.32 \mathrm{E}-02$ & $1.16 \mathrm{E}-01$ & -- & $1.41 \mathrm{E}-01$ \\
\hline 0.3201 & 7.91E-02 & $1.12 \mathrm{E}-01$ & -- & $1.44 \mathrm{E}-01$ & 0.3201 & $7.90 \mathrm{E}-02$ & $1.10 \mathrm{E}-01$ & -- & $1.41 \mathrm{E}-01$ \\
\hline 0.514 & $6.38 \mathrm{E}-02$ & $9.29 \mathrm{E}-02$ & -- & $1.07 \mathrm{E}-01$ & 0.514 & $6.38 \mathrm{E}-02$ & $9.10 \mathrm{E}-02$ & -- & $1.04 \mathrm{E}-01$ \\
\hline 0.6616 & $5.61 \mathrm{E}-02$ & $8.32 \mathrm{E}-02$ & - & $8.37 \mathrm{E}-02$ & 0.6616 & $5.59 \mathrm{E}-02$ & $8.15 \mathrm{E}-02$ & - & 8.07E-02 \\
\hline 1.115 & 4.04E-02 & $6.49 \mathrm{E}-02$ & -- & $5.64 \mathrm{E}-02$ & 1.115 & 4.03E-02 & $6.36 \mathrm{E}-02$ & -- & $6.23 \mathrm{E}-02$ \\
\hline 1.173 & $3.85 \mathrm{E}-02$ & $6.35 \mathrm{E}-02$ & -- & $6.93 \mathrm{E}-02$ & 1.173 & $3.89 \mathrm{E}-02$ & $6.22 \mathrm{E}-02$ & -- & $7.10 \mathrm{E}-02$ \\
\hline 1.333 & $3.56 \mathrm{E}-02$ & $5.94 \mathrm{E}-02$ & -- & $5.92 \mathrm{E}-02$ & 1.333 & $3.56 \mathrm{E}-02$ & $5.82 \mathrm{E}-02$ & -- & $5.84 \mathrm{E}-02$ \\
\hline
\end{tabular}

\section{Results and Discussion}

The simulated MCNPX, theoretical XCOM, other simulations (Geant4 and MCNP) along with possible experimental results of mass attenuation coefficient $(\mu / \rho)$ values for different photon energies are given in Table 1 (a)-(b). In general, it was found that the $\mu / \rho$ values for the concretes and dosimetric materials were very close to theoretical XCOM data, experimental results and other simulations. The slightly higher deviation in results is noted in the present investigation as compared with previous simulations. The possibility for deviation in the results may be the cross section files or computer features. The $\mu / \rho$ values calculated by MCNPX for dosimetric materials containing barium $\left(\mathrm{BaSO}_{4}\right.$ and $\left.\mathrm{C}_{4} \mathrm{H}_{4} \mathrm{BaO}_{4}\right)$ were found to be slightly higher than the remaining results for all the selected photon energies. The discrepancies in the $\mu / \rho$ values in present simulation and previous investigations could be due to more precise arrangement for high-atomic number element in the experimental set-up. However, at intermediate and high energies the MCNPX results were found in good agreement with theoretical XCOM data, experiment results and other simulation results. The $\mu / \rho$ values for Bakelite and concrete using MCNPX and Fluka [25] were also found comparable. It can be concluded that mass attenuation coefficients for compound or composite materials having low-as well as high-atomic number elements for low- to high-energy of photons are found comparable with the experiment and Geant-4, MCNP and Fluka simulation codes.

\section{Conclusion}

The MCNPX was used for simulation of mass attenuation coefficients for the concretes, results were found to be comparable with the theoretical XCOM values and experimental data. It can be concluded that mass attenuation 
coefficients for compound or composite materials having low-as well as high- atomic number elements for low- to high-energy of photons are found comparable with the experiment and Geant- 4 , MCNP and Fluka simulation codes. The present geometry can be used as standard geometry for MCNPX simulation for low- as well as highatomic number element materials. The present study would very useful for alternate gamma-ray interaction simulation tool for various energies for radiation dosimetry, medical and nuclear technology.

\section{References}

[1] J.H. Hubbell, Phys. Med. Biol. 51, R245 (2006). https://doi.org/10.1088/0031-9155/51/13/R15

[2] M.E. Medhat and Y. Wang, Annals of Nuclear Energy 62, 316 (2013). https://doi.org/10.1016/j. anucene.2013.06.034

[3] V.P. Singh and N.M. Badiger, Nuclear Technology and Radiation Protection 28, 137 (2013). https://doi. org/10.2298/NTRP1302137S

[4] V.P. Singh and N.M. Badiger, Radioprotection 48, 431 (2013). https://doi.org/10.1051/radiopro/2013067

[5] V.P. Singh and N.M. Badiger, Annals of Nuclear Energy 64, 301 (2013). https://doi.org/10.1016/j. anucene.2013.10.003

[6] V.P. Singh, A.M. Ali, N.M. Badiger and A.M. El-Khayatt, Nuclear Engineering and Design 265, 1071 (2013). https://doi.org/10.1016/j. nucengdes.2013.10.008

[7] V.P. Singh, M.E. Medhat and N.M. Badiger, Journal of Radioanalytical and Nuclear Chemistry 300, 325 (2014). https://doi.org/10.1007/s10967-014-2984-6

[8] Sh. Sharifi, R. Bagheri and S.P. Shirmardi, Annals of Nuclear Energy 53, 529 (2013).

https://doi.org/10.1016/j.anucene.2012.09.015

[9] S.P. Shirmardi, M. Shamsaei and M. Naserpour, Annals of Nuclear Energy 55, 288 (2013). https://doi. org/10.1016/j.anucene.2013.01.002

[10] A.M. El-Khayatt, A.M. Ali and V.P. Singh, Nuclear Instruments and Methods in Physics Research Section A: Accelerators, Spectrometers, Detectors and Associated Equipment 735, 207 (2013). https://doi. org/10.1016/j.nima.2013.09.027

[11] A.M. El-Khayatt, A.M. Ali, V.P. Singh and N.M. Badiger, Radiation Effects \& Defects in Solids 169,
1038 (2014). https://doi.org/10.1080/10420150.201 4.988626

[12] V.P. Singh, M.E. Medhat and N.M. Badiger, Annals of Nuclear Energy 68, 96 (2014). https://doi. org/10.1016/j.anucene.2014.01.011

[13] RSICC Computer Code Collection. MCNPX User's manual Version 2.4.0. Monte Carlo N-Particle Transport Code System for Multiple and High Energy Applications (2002).

[14] H.O. Tekin, Science and Technology of Nuclear Installations 2016, 6547318 (2016). https://doi. org/10.1155/2016/6547318

[15] I. Akkurt, H.O. Tekin and A. Mesbahi, Acta Physica Polonica A 128, B332 (2015). https://doi. org/10.12693/APhysPolA.128.B-332

[16] H.O. Tekin and U. Kara, Journal of Communication and Computer 13, 32 (2016).

[17] H.O. Tekin, T. Manici and C. Ekmekci, Journal of Health Science 4, 131 (2016).

[18] H.O. Tekin, V.P. Singh, U. Kara, T. Manici and E.E. Altunsoy, CBU Journal of Science 12, 195 (2016). https://doi.org/10.18466/cbujos.15586

[19] H.O. Tekin, T. Manici and V.P. Singh, Journal of Polytechnic 19, 617 (2016).

[20] M.J. Berger, J.H. Hubbell, S.M. Seltzer, J. Chang, J.S. Coursey, R. Sukumar, D.S. Zucker and K. Olsen, XCOM: photon cross sections database, NIST standard reference database 8 (XGAM) (2010). https://dx.doi.org/10.18434/T48G6X

[21] L. Gerward, N. Guilbert, K.B. Jensen and H. Levring, Radiation Physics and Chemistry 71, 653 (2004). https://doi.org/10.1016/j.radphyschem.2004.04.040

[22] I.I. Bashter, Annals of Nuclear Energy 24, 1389 (1997). https://doi.org/10.1016/S0306-4549(97)00003-0

[23] S. Gowda, S. Krishnaveni, T. Yashoda, T.K. Umesh and R. Gowda, Pramana 63, 529 (2004). https://doi. org/10.1007/BF02704481

[24] Shivaramu, R. Vijayakumar, L. Rajasekaran and N. Ramamurthy, Radiation Physics and Chemistry 62, 371 (2001). https://doi.org/10.1016/S0969806X(01)00221-3

[25] N. Demir, U.A. Tarim, M.-A. Popovici, Z.N. Demirci, O. Gurler and I. Akkurt, Journal of Radioanalytical and Nuclear Chemistry 298, 1303 (2013). https://doi. org/10.1007/s10967-013-2494-y 




CHITKARA
Journal of Nuclear Physics, Material Sciences, Radiation and Applications

Chitkara University, Saraswati Kendra, SCO 160-161, Sector 9-C, Chandigarh, 160009, India

Volume 8, Issue 1

August 2020

ISSN 2321-8649

Copyright: [C 2020 Huseyin Ozan Tekin and V.P. Singh] This is an Open Access article published in Journal of Nuclear Physics, Material Sciences, Radiation and Applications (J. Nucl. Phy. Mat. Sci. Rad. A.) by Chitkara University Publications. It is published with a Creative Commons Attribution- CC-BY 4.0 International License. This license permits unrestricted use, distribution, and reproduction in any medium, provided the original author and source are credited. 